\title{
One-dimensional Mott localization of quantum vortices in Josephson-junction arrays
}

\author{
A. van Oudenaarden, B. van Leeuwen, M. P. M. Robbens, and J. E. Mooij \\ Department of Applied Physics and Delft Institute of Micro-electronics and Submicron-technology (DIMES), \\ Delft University of Technology, P.O. Box 5046, 2600 GA Delft, The Netherlands
}

(Received 27 October 1997)

\begin{abstract}
The quantum transport of vortices in very long and narrow arrays of small Josephson junctions is studied experimentally. We investigate the forming of a Mott insulator in periodic arrays. When the vortex density is commensurate with the one-dimensional periodic potential, the vortices localize due to the strong repulsive vortex-vortex interaction. We explore the influence of interaction strength, bandwidth, sample geometry, and temperature on the stability of the Mott states. [S0163-1829(98)01018-2]
\end{abstract}

\section{INTRODUCTION}

Noninteracting quantum-mechanical particles in an ideal periodic potential are always delocalized. The solution of the Schrödinger equation consists of Bloch waves that extend through the system. Repulsive interaction can drive the particles into an insulating state: a Mott insulator. ${ }^{1}$ When the particle density is commensurate with the underlying periodic potential the particles form a Mott insulator, whereas for incommensurate filling a conducting phase exists for electrons and a superfluid phase for bosons. Onedimensional Josephson-junction arrays are very suitable systems to explore Mott localization ${ }^{2}$ of bosons in one dimension. Josephson-junction arrays are also used to probe quantum phase transitions in two dimensions. ${ }^{3}$ One of the first theoretical studies on boson localization by Fisher $e t$ al. ${ }^{1}$ calculated the mean-field phase diagram of bosons with short-range repulsive interaction revealing the Mott insulating phase for commensurate filling, a superfluid phase for incommensurate filling and an insulating Bose glass phase in the presence of sufficiently strong disorder. In more recent theoretical studies on one-dimensional bosons on a lattice the phase diagram is calculated by analytical ${ }^{4}$ or numerical means. ${ }^{5}$

A Josephson-junction array consists of a regular twodimensional lattice of superconducting islands. In a square array each island is weakly coupled to its four neighboring islands by Josephson junctions. At small magnetic fields, applied perpendicular to the plane of the array, circulating supercurrents, which are called vortices, minimize the total energy of the array. This effect is analogous to vortices in type-II superconductors. An important difference however is the absence of a nonsuperconducting core for a vortex in a Josephson-junction array. The motion of a vortex through a Josephson-junction array is homologous to the motion of a mass-carrying particle through a periodic potential. To travel from one minimum to the next in the periodic potential, the vortex has to overcome an energy barrier which is proportional to the Josephson coupling energy $E_{J}=i_{c 0} \Phi_{0} / 2 \pi$, where $i_{c 0}$ is the critical current of a single Josephson junction and $\Phi_{0}$ is the superconducting flux quantum. The other important energy scale is the charging energy $E_{C}=e^{2} / 2 C$, where $C$ is the capacitance of a junction and $e$ is the charge of an electron. In a quasistatic approximation ${ }^{6}$ the mass of a vortex $m_{v}$ is inversely proportional to the charging energy: $m_{v}=\Phi_{0}^{2} C /\left(2 a^{2}\right)$, where $a$ is the lattice constant of the array. The magnetic field applied perpendicular to the plane of an array plays the role of the chemical potential, which determines the density of vortices. The great advantage of a Josephson-junction array is that both the critical current and the capacitance, and therefore also the barrier height and the vortex mass, can be controlled accurately and can be varied independently over a large range. Within an array, which consists typically of more than $10^{4}$ junctions, the deviation in junction parameters can be made smaller than $5 \%$.

In the limit $E_{J} \gg E_{C}$ the vortex has a large mass and has to overcome high well-to-well barriers. In this limit the vortex behaves as a classical particle. The particle nature of the vortex is for example reflected in its ability to move ballistically ${ }^{7}$ or to reflect at boundaries. ${ }^{8}$ In the case of lower barriers and smaller vortex mass $\left(E_{J} \approx E_{C}\right)$ the vortex behaves as a quantum particle. ${ }^{6}$ In this regime the vortex behaves like a wave rather than a particle. The quantum vortex is able to tunnel, ${ }^{6}$ to interfere ${ }^{9}$ or to localize. ${ }^{2,10}$ In an ordered periodic array a quantum vortex experiences a periodic potential and will therefore form energy bands. In a nearly free vortex approximation the bandwidth is close to twice the charging energy $E_{C}$. The vortices repel each other over a long-range with a force which is proportional to $E_{J}$. By varying $E_{C}$ and $E_{J}$ the bandwidth and the interaction strength of the quantum vortices are changed, respectively. Unfortunately by varying $E_{J}$ not only the interaction strength between the vortices, but also the barrier height of the periodic potential is altered.

In this paper we present transport measurements on quantum vortices in one-dimensional Josephson-junction arrays. These systems are model systems for interacting bosons in a one-dimensional periodic potential. The sample layout, the fabrication procedure and details concerning the measurement setup are discussed in Sec. II. A model describing the forces on a vortex in a vortex lattice, which determines the interaction strength, is presented in Sec. III. In Sec. IV of the paper we explore the Mott transition due to the vortex-vortex interaction in ordered arrays. The influence of the interaction strength between the vortices (proportional to $E_{J}$ ) with respect to the bandwidth (proportional to $E_{C}$ ) on the Mott insulating phase is studied. We also compare Mott states in samples with different lengths and widths. By measuring the 


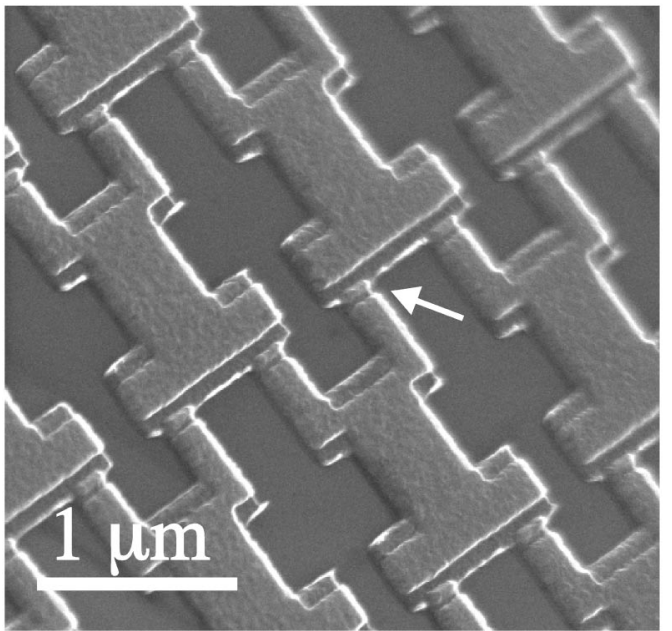

FIG. 1. Scanning electron micrograph of a uniform array. The arrow denotes a junction with an overlap area of $200 \times 100 \mathrm{~nm}^{2}$.

thermally activated transport in the Mott states, we deduce the Mott gap. Finally we summarize the experimental results in Sec. V.

\section{ONE-DIMENSIONAL JOSEPHSON-JUNCTION ARRAYS}

The samples consist of a square network of Josephson junctions in which each superconducting island is coupled to its four neighboring islands. In Fig. 1 a scanning electron microscope photograph of a detail of an array is shown. Using a shadow evaporation technique we fabricated highquality $\mathrm{Al}-\mathrm{Al}_{2} \mathrm{O}_{3}-\mathrm{Al}$ Josephson junctions. An example of such an overlap junction is denoted by the arrow in Fig. 1. The width of this junction is $200 \mathrm{~nm}$. The overlap area is about $200 \times 100 \mathrm{~nm}^{2}$, which corresponds to a capacitance $C$ of $2 \mathrm{fF}$. The mask of the arrays was defined using electron beam lithography and a two layer resist technique.

To confine the vortex motion to one dimension we designed the sample shown in Fig. 2(a). The length $L$ of the array is much larger than the width $W$. We measured samples with $L=100,200,300$ and 1000 cells and $W=3$ and 7 cells. Along the length of the array the islands at both edges are connected by a superconducting strip (busbar). The vortices are repelled by the busbar and therefore confined to one dimension. By injecting a current from busbar to busbar a force perpendicular to the current direction is exerted on the vortices. If this force is large enough the vortices start to move and induce a Josephson voltage across the array, which is measured at one end of the busbars [Fig. 2(a)].

For a uniform array [Fig. 2(a)] the vortices experience a periodic potential [Fig. 2(b)]. We calculated the height of the cell-to-cell barrier for a vortex moving along the middle row, denoted by the arrow in Fig. 2(a). The total potential energy $E=\Sigma_{i} E_{J}\left(1-\cos \phi_{i}\right)$ was computed iteratively by solving Kirchhoff's law at each island. $E_{J}$ is the Josephson coupling energy and $\phi_{i}$ is the gauge-invariant phase difference across junction $i$. Quantum fluctuations of the phase due to $E_{C}$ are not taken into account in this model. We neglect self-induced magnetic fields. This is reasonable, because the induced flux per cell $\Phi_{i} \approx L_{s} i_{c 0}$ is more than four orders smaller than $\Phi_{0}$.
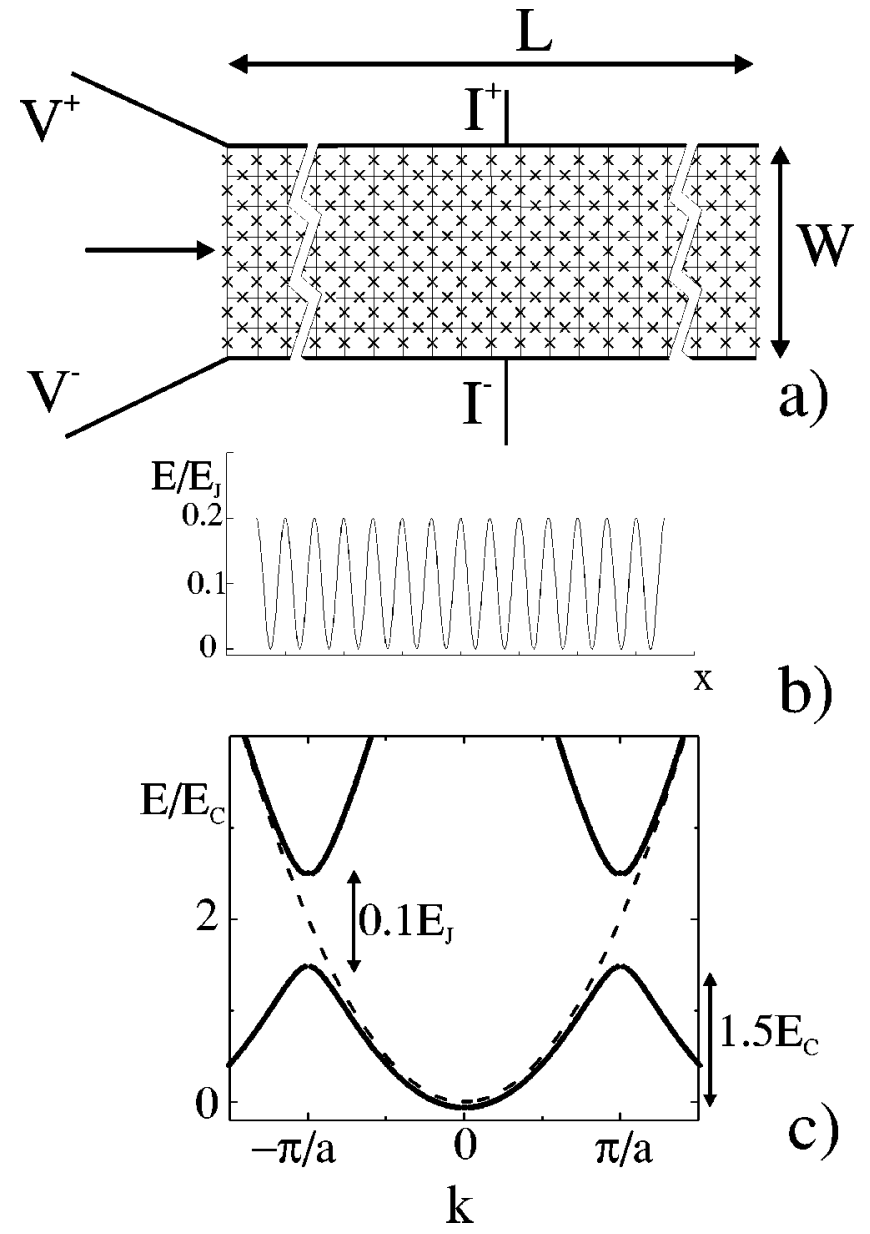

FIG. 2. (a) Sketch of the sample layout of an ordered onedimensional Josephson-junction array with length $L$ and width $W$. The crosses denote Josephson junctions. The current is injected in the middle of the busbars and the voltage probes are situated at one end of the busbars; (b) Potential energy versus position along the middle row; (c) Energy bands for a quantum vortex in a periodic potential $\left(E_{J} / E_{C}=10\right)$

The geometrical self-inductance $L_{s}$ is approximately equal to $\mu_{0} a$, where $\mu_{0}$ is the permeability of vacuum. The result of the computed potential is shown in Fig. 2(b). The barrier height is approximately $0.2 E_{J}$ (both for $W=3$ and $W=7$ ), which is close to the result obtained for an infinite twodimensional array. ${ }^{11}$ We also estimated the vortex mass in the one-dimensional geometry by calculating the phase differences when a vortex is moved over one cell. The computed mass is 33\% larger for $W=3$ and only 5\% larger for $W=7$ compared to the vortex mass in an infinite twodimensional system, which is given by $m_{v}=\Phi_{0}^{2} C /\left(2 a^{2}\right)$.

Using this static mass and a cell-to-cell barrier of $0.2 E_{J}$ we calculated the band structure of the quantum vortex by solving the Schrödinger equation for a vortex in a sinusoidal potential. For such a potential the Schrödinger equation reduces to Mathieu's equation, which can be solved analytically. ${ }^{12}$ The result for $E_{J} / E_{C}=10$ is shown in Fig. 2(c). The dashed line denotes the result for free quantum vortices. The parabola intersects the boundary of the first Brillouin zone at $2 E_{C}$. In a nearly-free vortex approximation the bandwidth $E_{0}$ is given by $E_{0}=\alpha E_{C}$, where $\alpha=2$ for $E_{J} / E_{C}=0$ and $\alpha \approx 1.5$ for $E_{J} / E_{C} \approx 10$. The two lowest-lying 
energy bands are separated by an energy gap of $0.1 E_{J}$.

All samples were cooled down in a dilution refrigerator with a base temperature of $20 \mathrm{mK}$. Josephson-junction arrays are extremely sensitive to magnetic interference. For example, magnetic noise which induces one extra flux quantum in the sample $(\approx 1 \mathrm{mG})$ can change the resistance of the array by one order of magnitude. The arrays were therefore carefully screened inside a Mumetal cylinder, which is coated by a thick superconducting lead screen. Small magnetic fields were applied by two superconducting coils in the Helmholtz configuration. The sample was mounted in the plane between the two coils to obtain an optimally homogenous magnetic field over the entire sample. Electrical signals were filtered by microwave copper powder filters and $\mathrm{RC}$ filters at the temperature of the mixing chamber. At room temperature the electrical leads were additionally filtered by $\Pi$ filters.

All measurements were performed in the linear-response regime. The bias current, which exerts an external force on the vortices, was much smaller than the current needed to drag the vortices over the barriers. In the linear-response regime the two remaining transport mechanisms are either quantum-mechanical tunneling through the barriers or thermal activation over the barriers. The zero-bias resistance $R_{0}$ was measured using a lock-in technique.

\section{FORCES ON A VORTEX IN A VORTEX LATTICE}

In this section we present a model that describes the forces on a vortex in a vortex lattice, which determines the interaction strength between the vortices. In the limit of large Josephson coupling energy $E_{J}$ with respect to the charging energy $E_{C}$ a Josephson-junction array is well characterized by the gauge-invariant phase differences $\phi$ between the islands. As discussed in the previous section the vortices preferably travel along the middle row of the array, denoted by the arrow in Fig. 2(a). In the following we therefore only consider the supercurrents flowing through the junctions that cross the middle row. These currents predominantly determine the repelling forces between the vortices. The $x$ and $y$ coordinates are chosen parallel and perpendicular to the busbars, respectively. In Fig. 3(a) the current distribution is shown for an array $(W=7 ; L=100)$ with one vortex in the middle $(x / a=50)$ in the presence of a small magnetic field. We computed this distribution by minimizing the total potential energy of the array $E=\Sigma_{i} E_{J}\left(1-\cos \phi_{i}\right)$. We choose the gauge of the magnetic vector potential $\mathbf{A}$ such that phase differences along the busbars due to $\mathbf{A}$ are zero: $\mathbf{A}=$ $-B(x / a) \hat{\mathbf{y}}$, where $\hat{\mathbf{y}}$ is the unit vector in the $y$ direction which is perpendicular to the busbars and $B$ is the magnetic field. For this particular gauge it is instructive to split the gauge-invariant phase difference $\phi_{i}$ across junction $i$ in a part $\phi_{A}$, which is determined by the magnetic vector potential $\mathbf{A}$, and a term $\phi_{V}$, which reflects the presence of vortices. The phase difference between island $i$ and $j$ due to the magnetic vector potential is given by $\phi_{A}=\left(2 \pi / \Phi_{0}\right) \int_{i}^{j} \mathbf{A}$ -dl. This leads to $\phi_{A}=-2 \pi f(x / a)$, where the frustration $f$ is the magnetic flux through a cell of the array normalized to $\Phi_{0}$. A more relevant quantity for the one-dimensional system is the one-dimensional frustration $n$, which is the magnetic flux through a column of $W$ cells: $n=W f$. In the ab-

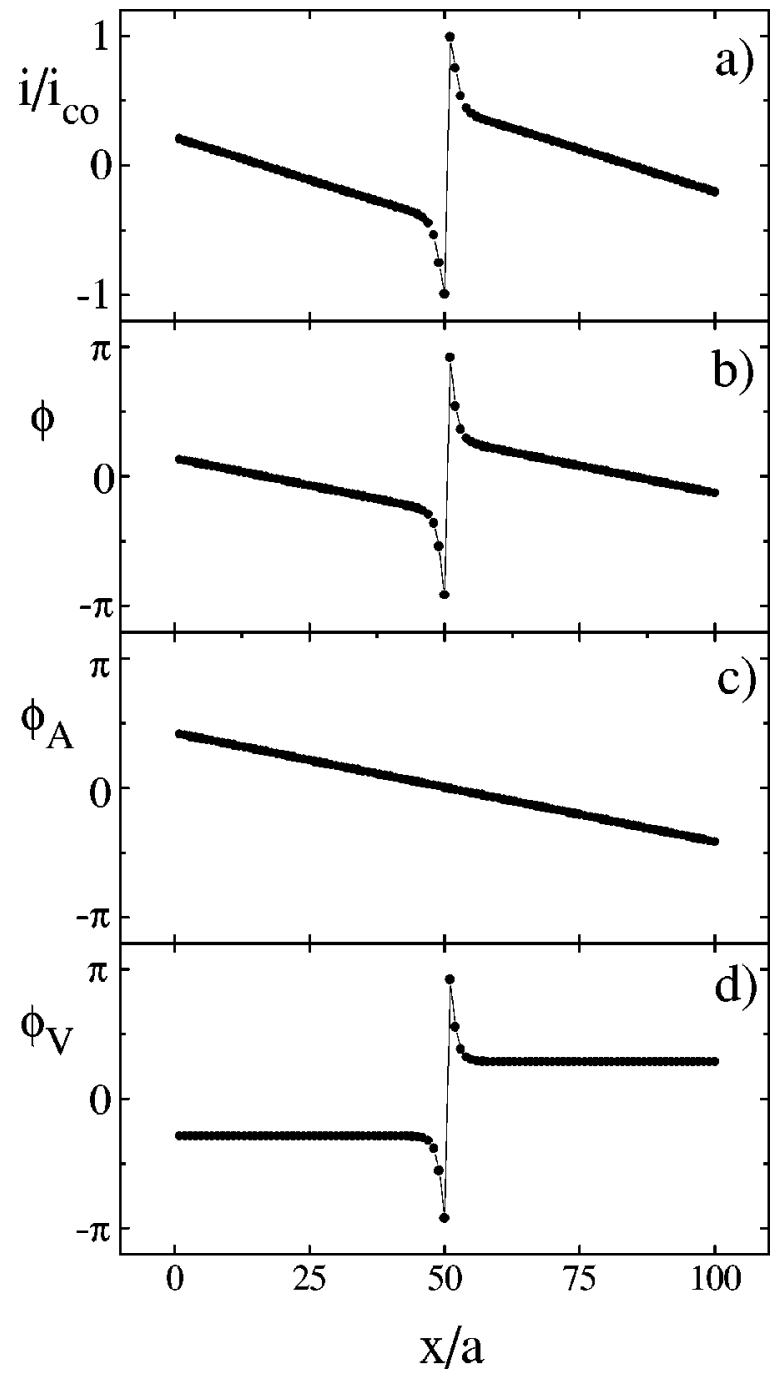

FIG. 3. (a) Current distribution in an array $(W=7 ; L=100)$ with one vortex in the middle of the array $(f=0.0021)$; (b) Gaugeinvariant phase difference $\phi=\phi_{A}+\phi_{V}$ across the middle row of the array; (c) Phase difference due to the magnetic vector potential; (d) Phase difference which is independent of the magnetic vector potential. The phase step defines the vortex.

sence of a periodic potential [Fig. 2(b)] the vortices form an equidistant vortex lattice with a period $1 / n$. In Fig. 3(a) the supercurrent $i(x)=i_{c 0} \sin \phi(x)$ is shown as a function of $x$ for $f=0.0021$, where $i_{c 0}$ is the critical current of a junction. The corresponding gauge-invariant phase difference $\phi$ [Fig. 3(b) ] can now be split in $\phi_{A}$ and $\phi_{V}$, shown in Figs. 3(c) and $3(d)$, respectively. The contribution of a vortex is reflected in a step of $\phi_{V}$ at the position of the vortex. Far left and right from the vortex center $\phi_{V}$ equals $-\pi / W$ and $+\pi / W$, respectively. When $f$ is increased $\phi_{A}$ becomes a steeper function. At a certain frustration it is more favorable to allow an extra vortex in the array, which results in an extra step in $\phi_{V}$. The step reduces the gauge-invariant phase differences and the total energy. A vortex in the middle of the array, which has an equal number of vortices on the left and the right, feels a resultant force $F(x)=i(x) \Phi_{0} / a \approx \phi_{A}(x) \Phi_{0} / a$ due to the magnetic vector potential. This force leads to a parabolic potential $U(x)=2 \pi^{2} f E_{J}(x / a)^{2}=2 \pi^{2} n E_{J}(x / a)^{2}$ / $W$. An equivalent result is obtained for vortices which have 
TABLE I. Sample parameters of the uniform one-dimensional arrays used for the Mott localization measurements.

\begin{tabular}{|c|c|c|c|c|c|c|c|}
\hline$L$ & $W$ & $\begin{array}{l}R_{n} \\
(\Omega)\end{array}$ & $\begin{array}{c}r_{n} \\
(\mathrm{k} \Omega)\end{array}$ & $\begin{array}{c}C \\
(\mathrm{fF})\end{array}$ & $\begin{array}{c}E_{J} / k_{B} \\
(\mathrm{~K})\end{array}$ & $\begin{array}{c}E_{C} / k_{B} \\
(\mathrm{~K})\end{array}$ & $E_{J} / E_{C}$ \\
\hline 1000 & 7 & 39.2 & 5.6 & 2.1 & 1.24 & 0.4 & 2.8 \\
\hline 1000 & 7 & 53.2 & 7.6 & 1.5 & 0.91 & 0.7 & 1.3 \\
\hline 1000 & 7 & 77.7 & 11.1 & 1.1 & 0.62 & 0.9 & 0.7 \\
\hline 1000 & 3 & 29.3 & 9.7 & 2.0 & 0.71 & 0.5 & 1.5 \\
\hline 100 & 7 & 708 & 10.1 & 2.0 & 0.69 & 0.5 & 1.5 \\
\hline 200 & 7 & 343 & 9.8 & 2.0 & 0.71 & 0.5 & 1.5 \\
\hline 300 & 7 & 211 & 9.0 & 2.0 & 0.77 & 0.5 & 1.7 \\
\hline
\end{tabular}

a different number of vortices on the left and the right. This approximation is only valid if the vortex cores, for which $\left|\phi_{V}\right|>\pi / W$, do not overlap. The energy $U(x)$ determines the interaction strength, which can be varied by changing both $E_{J}$ or $n$. The vortices also feel the periodic potential with height $0.2 E_{J}$, as discussed in the previous section. At $x=\frac{1}{2} a$ the two energies are equal for the one-dimensional density $n^{*} \approx 0.4 W / \pi^{2}$. In the limit $n \ll n^{*}$ the parabolas are very shallow and locally the periodic potential is much stronger than the forces in the vortex lattice. The vortices will always occupy energy minima of the periodic potential independent of the small forces between the vortices in the vortex lattice. In this limit no Mott insulator can exist. However in the limit of $n \gg n^{*}$ the vortices will form a rigid vortex lattice which for commensurate densities collectively pins to the junction lattice. The crossover density $n^{*}$ equals 0.12 for $W=3$ and 0.28 for $W=7$.

In the model the vortex-vortex interaction is infinitely long range, because induced magnetic fields are completely neglected. For a two-dimensional array ${ }^{13}$ the range over which vortices interact, is estimated by $\lambda_{2 \mathrm{D}}$ $=\Phi_{0} /\left(2 \pi \mu_{0} i_{c 0} a\right)$. For our arrays with $E_{J} \approx E_{C}, \lambda_{2 \mathrm{D}}$ is of the order of $10^{3}$ lattice cells, which is of the order of $L$. This means that the vortices in our arrays interact over a long range and that effects due to the inductance are negligible.

\section{MOTT LOCALIZATION}

In this section we present the experimental results on seven uniform samples, for which the sample layout is sketched in Fig. 2(a). We varied the $E_{J} / E_{C}$ ratio to examine the effect of the bandwidth and the interaction strength on the Mott insulating state. We also fabricated samples with different lengths and widths to study the effect of the geometry. The properties of the samples are summarized in Table I. The normal-state array resistance $R_{n}$ equals $W r_{n} / L$, where $r_{n}$ is the normal-state junction resistance. The Josephson coupling energy $E_{J}=i_{c 0} \Phi_{0} / 2 \pi$ is determined using the Ambegaokar-Baratoff result for aluminum with a critical temperature $T_{c}=1.2 \mathrm{~K}: i_{c 0} r_{n}=322 \mu \mathrm{V}$. The charging energy $E_{C}=e^{2} / 2 C$ is estimated using the overlap area of the junctions. An overlap of $100 \times 100 \mathrm{~nm}^{2}$ corresponds to a capacitance $C$ of approximately $1 \mathrm{fF}\left(E_{C} / k_{B} \approx 0.9 \mathrm{~K}\right)$.

The vortex density in a Josephson-junction array is changed by an external magnetic field $B$. The twodimensional frustration $f$ is defined as the applied magnetic

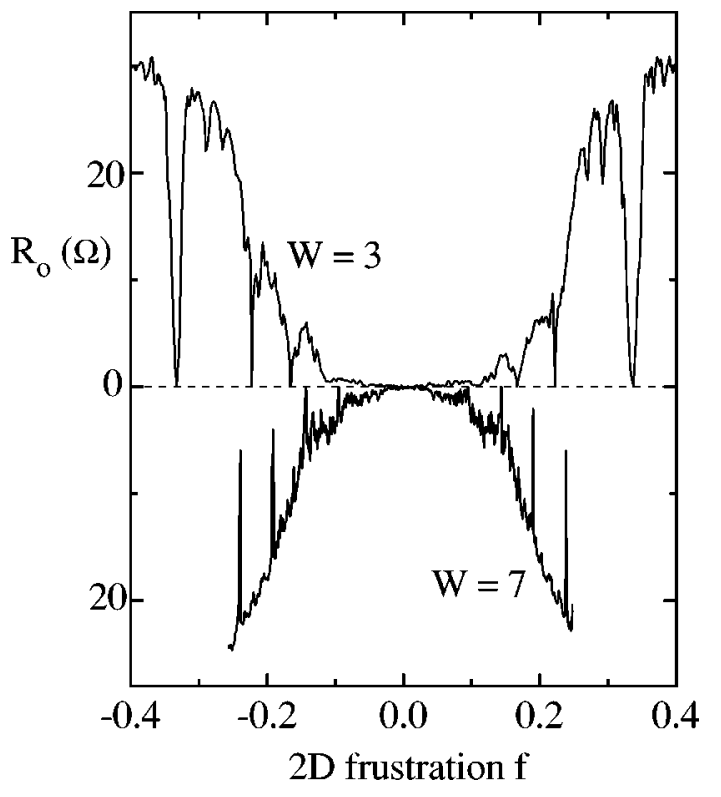

FIG. 4. Zero-bias resistance $R_{0}$ versus two-dimensional frustration $f$ for two samples with different widths $W(L=1000)$. The curve of the $(W=7)$ sample is mirrored with respect to the $x$ axis for clarity $(T=30 \mathrm{mK})$.

flux $B S$ per cell area divided by $\Phi_{0}$, where $S$ is the area of a cell which is $2 \mu \mathrm{m}^{2}$. If $f=1$ every cell in the array contains one flux quantum. In Fig. 4 the zero-bias resistance $R_{0}$ is plotted as a function of the two-dimensional frustration $f$ for two samples with different $W$. The plot of the $(W=7)$ sample is mirrored with respect to the $x$ axis for clarity. For both samples distinct sharp dips are observed at certain values of $f$. For $W=3$ sharp dips at $f=1 / 6,2 / 9,1 / 3$, and $4 / 9$ are observed. For a wider array $(W=7)$ we observe dips at $f=1 / 21,2 / 21$, and 3/21. In Fig. 5 we plotted the same results

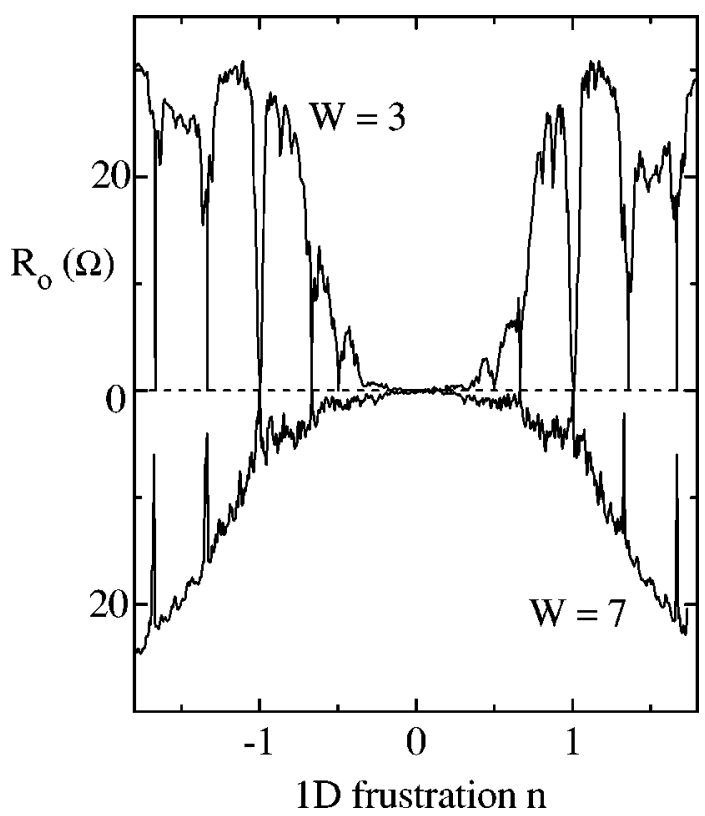

FIG. 5. Zero-bias resistance $R_{0}$ versus one-dimensional frustration $n=W f$ for two samples with different widths $W(L=1000)$. The curve of the $(W=7)$ sample is mirrored with respect to the $x$ axis for clarity $(T=30 \mathrm{mK})$. 


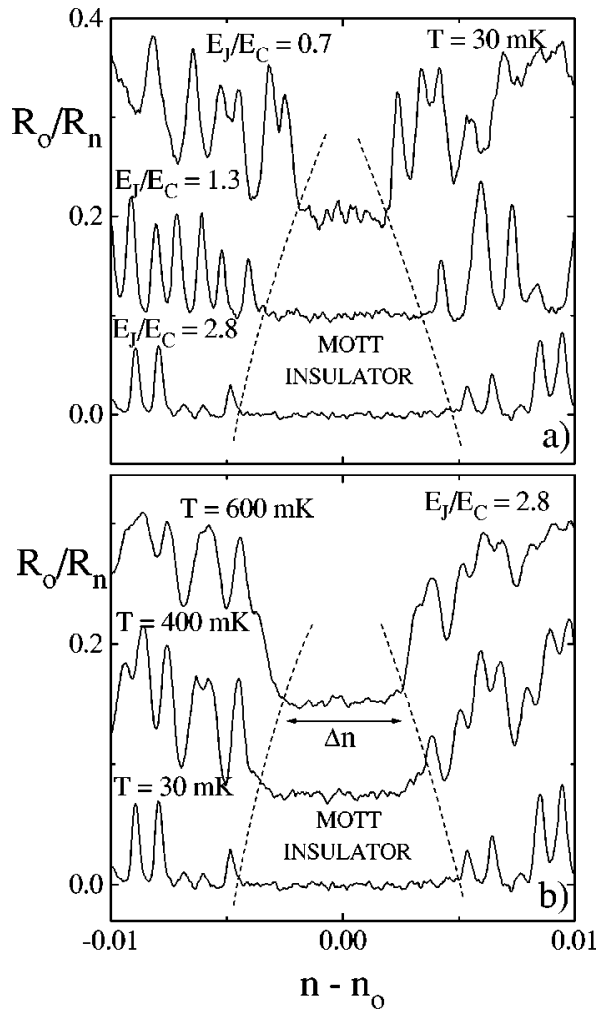

FIG. 6. (a) Normalized zero-bias resistance $R_{0} / R_{n}$ at $T$ $=30 \mathrm{mK}$ versus $n-n_{0}$ around the one-dimensional commensurate filling $n_{0}=1 / 3$ for samples with different $E_{J} / E_{C}(T=30 \mathrm{mK})$. The two upper curves have an offset in $R_{0} / R_{n}$ of 0.1 and 0.2 . The dashed line indicates the phase boundary, which separates the Mott insulating phase from the conducting phase. (b) Normalized zerobias resistance $R_{0} / R_{n}$ versus $n-n_{0}$ at different temperatures for $E_{J} / E_{C}=2.8$.

but now as a function of the one-dimensional frustration $n$, which is defined as the magnetic flux WBS through a onedimensional cell, which has an area of $W S$, divided by $\Phi_{0}$, hence $n=W B S / \Phi_{0}=W f$. The sharp dips in $R_{0}$ are observed at the same values of $n$ for both samples and the dips are observed around fractions of $n$, such as $n=1 / 3,1 / 2,1,2$. This demonstrates the one-dimensional origin of the observed effect. In the remaining part of this section we interpret this strong decrease of the zero-bias resistance around rational numbers of the one-dimensional frustration in terms of the forming of a one-dimensional Mott insulator of vortices due to the repulsive vortex-vortex interaction.

In Fig. 6(a) the zero-bias resistance $R_{0}$ for three samples $(L=1000 ; W=7)$ with different $E_{J} / E_{C}$ ratios is shown as a function of $n-n_{0}$, where $n_{0}=1 / 3$. In these three samples we observed the same effect around $n_{0}=2 / 3$. From this figure it is apparent that the sharp dips in Fig. 5 are not infinitely sharp, but $R_{0}=0$ for a finite window of $n$. At a certain $n$ $-n_{0}, R_{0}$ increases sharply, which means that the vortex chain is depinned. In this conducting regime $R_{0}$ varies roughly periodically as a function of $n$ with a period $1 / L$ $=1 / 1000$. Each period corresponds to adding one extra vortex to the array. ${ }^{14}$ In the following we are explicitly interested in the insulating phase around one-dimensional commensurate filling. Around these fillings the vortices pin collectively to the periodic potential and form a Mott insula- tor $\left(R_{0}=0\right)$. The Mott insulator is stable for a finite region around $n-n_{0}=0$. In this region the interaction energy dominates the bandwidth. At a certain $n-n_{0}, R_{0}$ increases sharply. At this point the vortices delocalize $\left(R_{0} \neq 0\right)$ because the bandwidth starts to dominate the interaction energy. The forming of a Mott insulator of bosons with repulsive interaction is determined by the competition of two energies: the interaction energy and the bandwidth. ${ }^{1}$ In the limit of very strong interaction with respect to the bandwidth the bosons always form a Mott insulator. In the opposite limit the bosons are delocalized. The repelling force between two quantum vortices is directly proportional to $E_{J}$ and the bandwidth is directly proportional to $E_{C}$. By changing the $E_{J} / E_{C}$ ratio of an array the tendency to localize is altered. For samples with a relatively large $E_{J} / E_{C}$ ratio (large interaction energy compared to bandwidth) the vortices are localized for a larger range of $n-n_{0}$ than samples with a smaller $E_{J} / E_{C}$ ratio. In this way the lobelike shape of the phase diagram in Fig. 6(a) is determined experimentally. The Mott phase is incompressible, which means that although the chemical potential, i.e., $n-n_{0}$, is changed the total number of bosons, i.e., vortices, in the system remains constant. To destroy the Mott phase a particle or a hole should be created. The energy to create a particle or a hole in the vortex chain is called the Mott gap $E_{g}$. This gap has its maximum value at $n-n_{0}=0$ and $E_{g}=0$ at the phase boundary. The gap $E_{g}$ is also a function of $E_{J} / E_{C}$. For large interaction energies compared to bandwidth (large $E_{J} / E_{C}$ ) the Mott insulator is very stable and $E_{g}$ is large, whereas for small $E_{J} / E_{C}$ the Mott insulator is easily depinned, resulting in a small $E_{g}$. A similar interpretation involves the concept of a commensurate-incommensurate transition in the presence of quantum fluctuations. ${ }^{15}$ For finite $E_{C}$, the quantum fluctuations tend to renormalize (decrease) the amplitude of the periodic potential $\left(0.1 E_{J}\right)$. Therefore the region of a stable commensurate insulating phase is narrowed as $E_{J} / E_{C}$ is decreased.

In Fig. 6(b) $R_{0} / R_{n}$ is plotted versus $n-n_{0}$ for the sample with $E_{J} / E_{C}=2.8(L=1000, W=7)$ for different temperatures. At finite temperatures the thermal activation destroys the Mott states for which $E_{g}$ is comparable to $k_{B} T$. Experimentally this is reflected in a decrease of the range $\Delta n$ where the Mott insulator is stable $\left(R_{0}=0\right)$ as a function of temperature. At $T=0 \Delta n$ has its maximum value which is determined by $E_{J} / E_{C}$. When the temperature is raised, the first instabilities occur near the phase boundaries, because $E_{g}$ is smallest there. As a function of temperature the phase boundaries move towards $n_{0}$. This mechanism results in a decreasing $\Delta n$ as a function of $T$, which is observed experimentally in Fig. 6(b).

To verify if the array length is long enough to define a one-dimensional system and to study possible edge effects we fabricated samples with different lengths $(L=100,200$, and 300 cells). In Fig. $7 \Delta n$ is plotted versus $T$ for three samples with different $L$ and $W=7$ around $n_{0}=1 / 3$. The $E_{J} / E_{C}$ ratio of these samples is about 1.6. At low temperatures $\Delta n \approx 0.009$, this value is consistent with the experiments $(L=1000)$ shown in Fig. 6(a). The two samples with $L=200$ and $L=300$ show the same temperature dependence. However the $(L=100)$ sample is less stable and the Mott 


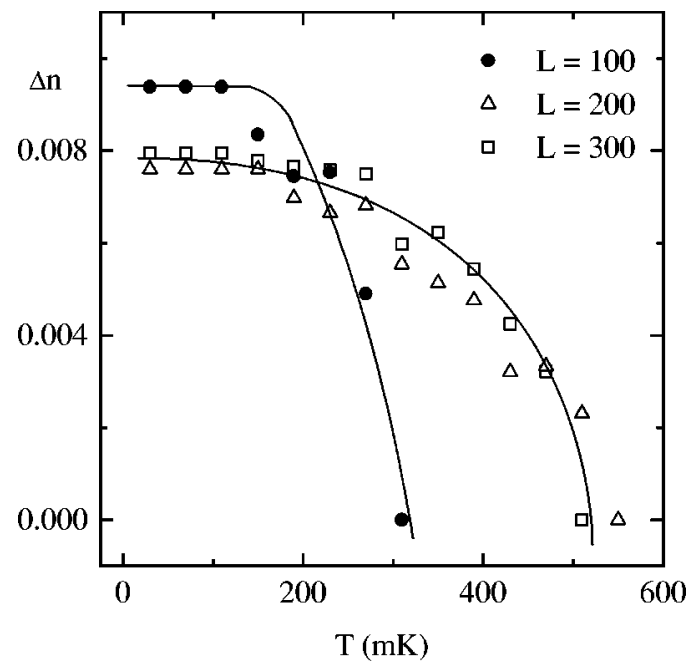

FIG. 7. The range $\Delta n$ for which the vortices form a Mott insulator as a function of temperature for three samples with different $L$ around the commensurate filling $n_{0}=1 / 3(W=7)$.

phase is completely destroyed at $T=300 \mathrm{mK}$, whereas the longer samples survive a temperature of $500 \mathrm{mK}$. Probably $L=100$ is not long enough to define a one-dimensional system and edge effects play a significant role. However for $L$ $\geqslant 200, \Delta n$ is independent of $L$ indicating that for these lengths the array is a well defined one-dimensional system.

In Fig. 8 the dependence of $n_{0}$ on the stability of the Mott phase is studied for the sample with $W=3$ and $L=1000$. It is apparent from this figure that for integer commensurate filling $n_{0}=1$ the Mott insulator is more stable than for halfinteger commensurate filling $n_{0}=1 / 2$. The distance between the vortices for the integer filling $n_{0}=1$ is smaller than the vortex-vortex distance for the half-integer filling $n_{0}=1 / 2$. Since the repelling forces between the vortices are significantly larger for $n_{0}=1$ than for $n_{0}=1 / 2$, the Mott state is more stable for integer filling. By varying $n_{0}$ the interaction

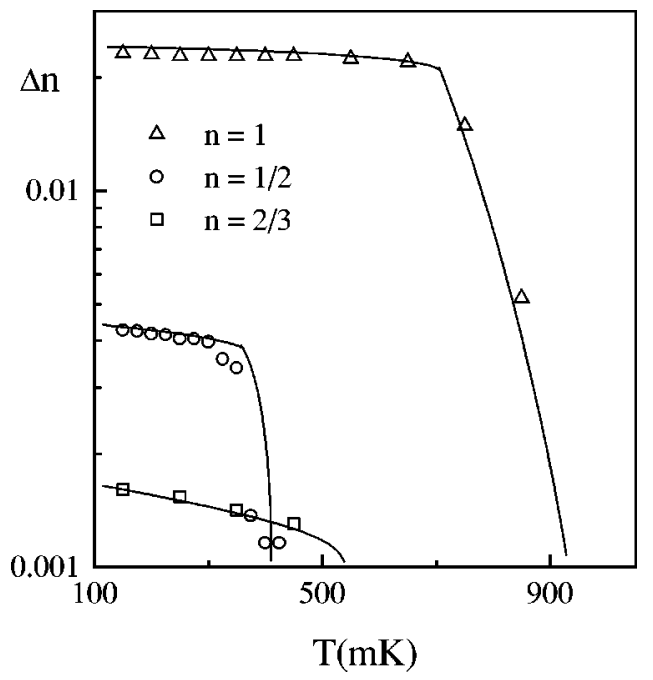

FIG. 8. The range $\Delta n$ for which the vortices form a Mott insulator as a function of temperature for three different commensurate fillings $(L=1000 ; W=3) . \Delta n$ is plotted on a logarithmic scale for clarity.

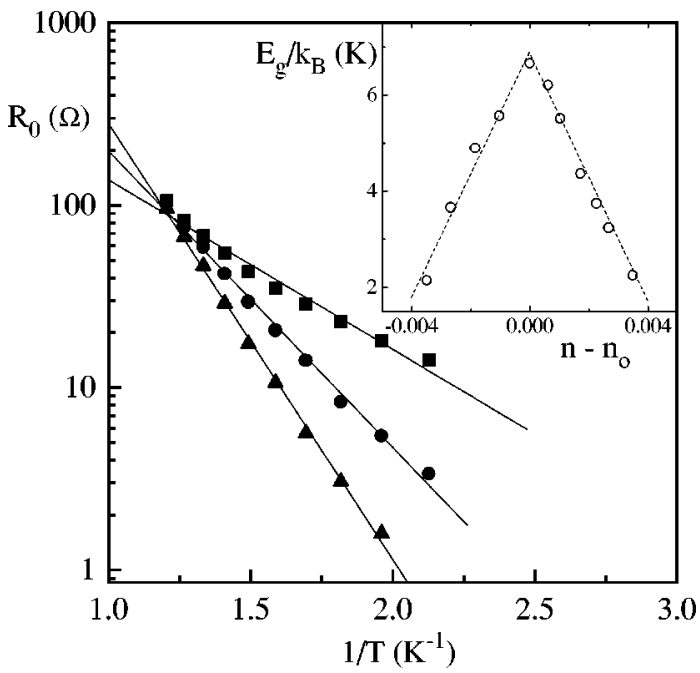

FIG. 9. Arrhenius plot of $R_{0}$ for $n-n_{0}=0.0010$ (triangles), $n$ $-n_{0}=0.0023$ (circles) and $n-n_{0}=0.0035$ (squares). From the slopes in the Arrhenius plot the Mott gap $E_{g}$ is deduced, which is plotted in the inset as a function of $n-n_{0} .(L=300 ; W=7)$.

strength is effectively altered. The Mott phase at $n_{0}=2 / 3$ is less stable than at $n_{0}=1 / 2$ and $n_{0}=1$. For $n_{0}=2 / 3$ an asymmetric vortex chain is formed for which the vortex-vortex distance is alternating between $a$ and $2 a$. Probably the asymmetry in the chain leads to smaller stability.

At finite temperature the mobility of the vortices in the Mott states has an activated form: $R_{0} \propto \exp \left(-E_{g} / k_{B} T\right)$. By measuring $R_{0}$ as a function of $T$ at different $n-n_{0}$, the Mott gap $E_{g}$ as a function of $n-n_{0}$ is deduced. In Fig. 9 an Arrhenius plot of $R_{0}$ for the ( $L=300$ ) sample is shown demonstrating the thermally activated behavior at elevated temperatures $(T>500 \mathrm{mK})$ for different values of $n-n_{0}$. From the slope $E_{g}$ is deduced and is plotted in the inset as a function of $n-n_{0}$. For $n-n_{0}=0$ the Mott gap has its maximum value. The Mott gap decreases linearly for increasing $\mid n$ $-n_{0} \mid$. The Mott gap for $L=200$ yields similar results. However the gap for $L=100$ is significantly smaller, which is consistent with the results shown in Fig. 7.

\section{CONCLUSION}

We have reported on experiments on vortices in onedimensional Josephson-junction arrays. These arrays are model systems for repulsively interacting bosons in one dimension. In periodic arrays localization of the quantum vortices was observed around one-dimensional commensurate values of the one-dimensional vortex density. This localization is due to the forming of a one-dimensional Mott insulator. We studied the influence of the bandwidth and the interaction strength on the stability of the Mott insulator. In this way we were able to construct the phase diagram for a onedimensional system experimentally. The influence of the length and width of the arrays was also studied. No significant difference was observed in arrays with length $L \geqslant 200$. By analyzing the thermally activated transport in the Mott states the Mott gap was deduced. 


\section{ACKNOWLEDGMENTS}

We want to thank A. A. Odintsov, A. van Otterlo, K.-H. Wagenblast, R. Fazio, H. S. J. van der Zant, and P. Hadley for useful discussions. The Delft Institute of Micro- electronics and Submicron-technology (DIMES) is acknowledged for the support in fabrication. The work was supported by the Dutch Foundation for Fundamental Research on Matter (FOM).
${ }^{1}$ M. P. A. Fisher, P. B. Weichman, G. Grinstein, and D. S. Fisher, Phys. Rev. B 40, 546 (1989).

${ }^{2}$ A. van Oudenaarden and J. E. Mooij, Phys. Rev. Lett. 76, 4947 (1996)

${ }^{3}$ H. S. J. van der Zant, F. C. Fritschy, W. J. Elion, L. J. Geerligs, and J. E. Mooij, Phys. Rev. Lett. 69, 2971 (1992); H. S. J. van der Zant, W. J. Elion, L. J. Geerligs, and J. E. Mooij, Phys. Rev. B 54, 10081 (1996).

${ }^{4}$ J. K. Freericks and H. Monien, Europhys. Lett. 26, 545 (1994); Phys. Rev. B 53, 2691 (1996).

${ }^{5}$ G. G. Batrouni, R. T. Scalettar, and G. T. Zimanyi, Phys. Rev. Lett. 65, 1765 (1990); P. Niyaz, R. T. Scalettar, C. Y. Fong, and G. G. Batrouni, Phys. Rev. B 50, 362 (1994), and references therein.

${ }^{6}$ E. Simanek, Solid State Commun. 48, 1023 (1983); S. E. Korshunov, Physica B 152, 261 (1988); A. I. Larkin, Yu. N. Ovchinikov, and A. Schmid, Physica B 152, 266 (1988); U. Eckern and A. Schmid, Phys. Rev. B 39, 6441 (1989).

${ }^{7}$ H. S. J. van der Zant, F. C. Fritschy, T. P. Orlando, and J. E.
Mooij, Europhys. Lett. 18, 343 (1992).

${ }^{8}$ T. J. Hagenaars, J. E. van Himbergen, J. V. José, and P. H. Tiesinga, Phys. Rev. B 53, 2719 (1996).

${ }^{9}$ W. J. Elion, J. J. Wachters, L. L. Sohn, and J. E. Mooij, Phys. Rev. Lett. 71, 2311 (1993).

${ }^{10}$ A. van Oudenaarden, S. J. K. Várdy, and J. E. Mooij, Phys. Rev. Lett. 77, 4257 (1996).

${ }^{11}$ C. J. Lobb, D. W. Abraham, and M. Tinkham, Phys. Rev. B 27, 150 (1983).

${ }^{12}$ Handbook of Mathematical Functions, edited by M. Abramowitz and I. A. Stegun (Dover, New York, 1965), p. 721.

${ }^{13}$ J. R. Phillips, H. S. J. van der Zant, J. White, and T. P. Orlando, Phys. Rev. B 47, 5219 (1993).

${ }^{14}$ A. van Oudenaarden, S. L. Yeung, and J. E. Mooij, in Macroscopic Quantum Phenomena and Coherence in Superconducting Networks, edited by C. Giovanella and M. Tinkham (World Scientific, Singapore, 1995).

${ }^{15}$ P. Bak and H. Fukuyama, Phys. Rev. B 21, 3287 (1980). 